Bull. Mater. Sci., Vol. 2, Number 3, August 1980, pp. 187-192. (C) Printed in India.

\title{
Exchange constants in ferrimagnetic garnets
}

\author{
C M SRIVASTAVA, C SRINIVASAN and R AIYAR \\ Department of Physics, Indian Institute of Technology, Bombay 400 076, India
}

MS received 9 April 1980

\begin{abstract}
The exchange constants in the ferrimagnetic garnets $\mathrm{R}_{3} \mathrm{Fe}_{5} \mathrm{O}_{1.2}\left(\mathrm{R}=\mathrm{Y}^{3+}\right.$, $\mathrm{Gd}^{3+}$ and $\mathrm{Dy}^{3+}$ ) have been obtained from the experimental data on saturation magnetisation and inverse susceptibility. The sign and magnitude of the exchange constants in $\mathrm{Y}_{3} \mathrm{Fe}_{3} \mathrm{O}_{12}$ have been explained on the basis of Anderson's theory of superexchange. The low temperature magnetisation data in $\mathrm{Dy}_{3} \mathrm{Fe}_{5} \mathrm{O}_{12}$ have been explained by assuming canting on the $c$ sublattice.
\end{abstract}

Keywords. Ferrimagnetic garnets; exchange constants; molecular field constants; transfer integrals; superexchange theory.

\section{Introduction}

The exchange constants in ferrimagnetic garnets have been obtained by many workers (Aléonard 1960; Dionne 1970; Dionne 1976; Anderson 1964) from either the magnetisation $\left(M_{s}\right)$ or the inverse susceptibility $\left(\chi^{-1}\right)$ data using the molecular field approximation, but the same set of exchange constants has not been used to fit both. We have attempted to fit both the $M_{3}$ and $\chi^{-1}$ data using a single set of exchange constants for the garnet systems, $\mathrm{Y}_{3} \mathrm{Fe}_{5} \mathrm{O}_{12}, \mathrm{Gd}_{3} \mathrm{Fe}_{5} \mathrm{O}_{12}$ and $\mathrm{Dy}_{3} \mathrm{Fe}_{5} \mathrm{O}_{12}$, using the molecular field approximation. This is important since it is found that the $M$, vs $T$ curves can be fitted with more than one set of exchange constants. This is also true in the case of the $\chi^{-1}$ vs $T$ curves. But of these only one set fits both the $M_{s}$ and $\chi^{-1}$ data and this set has been taken to be the true set of exchange constants. The sign and magnitude of the exchange constants have been explained on the basis of the Anderson's theory of super exchange (Anderson 1959). The data on YIG and GdIG could be fitted assuming collinear spin arrangement but in the case of DyIG the saturation magnetisation at $0 \mathrm{~K}$ calculated from the Néel model is different from that observed experimentally. This discrepancy has been attributed by Dionne (1976) to canting within the c-sub-lattice, which is assumed to arise from the strong anisotropy field of the $\mathrm{Dy}^{3+}$ ions as compared to the exchange field on the $c$-sub-lattice. However, his assumption of constant canting from $0 \mathrm{~K}$ to $T_{c}$ is not justified since both the exchange and anisotropy fields are temperature-dependent. We have shown that the canting angle falls rapidly with temperature and at about $30 \mathrm{~K}$ it vanishes and the system becomes Néel type. 


\section{Magnetic ordering and exchange constants}

We have used the method of calculating the exchange constants from the data on $M_{s}$ and $\chi^{-1}$ in systems with three collinear sub-lattices discussed elsewhere (Srivastava et al 1979). The substitution of magnetic rare earth ions in place of $\mathrm{Y}^{3+}$ in YIG is not expected to affect the exchange interactions between the transition metal ions in YIG. We have therefore used the same values of the $J_{a a}, J_{a d}$ and $J_{d d}$ for fitting the experimental data. The values of $J_{a s}, J_{d o}$ and $J_{n c}$ are one order of magnitude smaller but the susceptibility data is a sensitive function of these parameters.

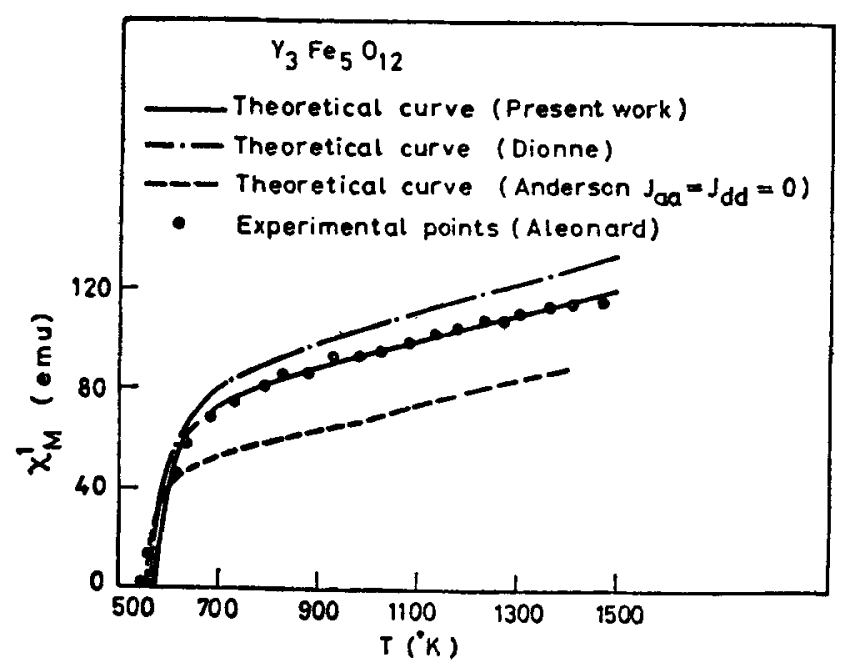

(a)

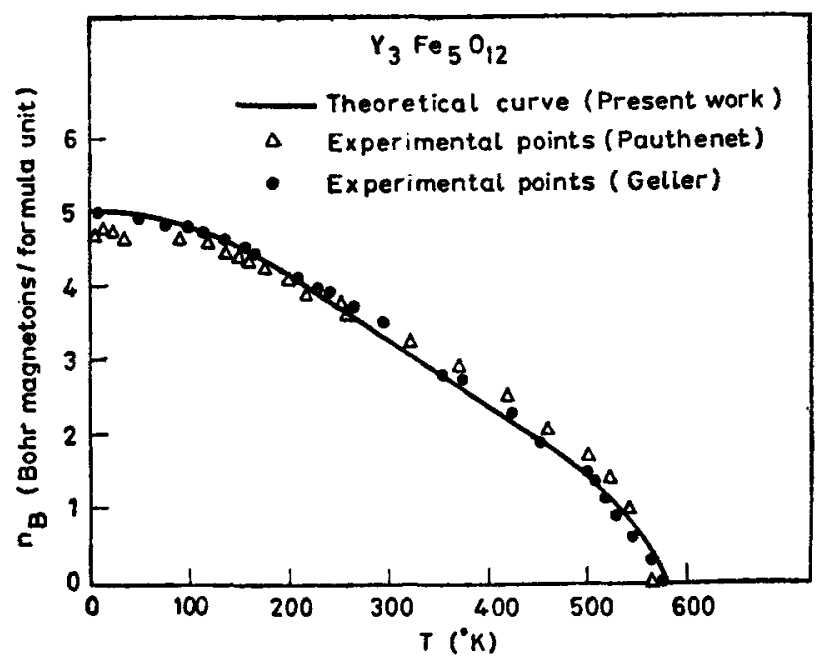

(b)

Figure 1. Theoretical and experimental curves for (a) saturation magnetisation $\left(\mathbf{M}_{s}\right)$ and (b) inverse susceptibility as a function of temperature for YTG. The theoretical curve has been calculated using the exchange constants listed in table 1. 
The theoretical curves for $M_{s}$ and $\chi^{-1}$ for YIG and GdIG are given in figures 1 and 2. The experimental points are from Aléonard (1960), Geller et al (1963, 1965) and Pauthenet (1957). The spin arrangement throughout the temperature range is Néel type.

In the case of DyIG the curve obtained on the collinear spin arrangement agrees with the experimental data only above $30 \mathrm{~K}$. Below this temperature the spin arrangement is canted, the canting angle varying from $45^{\circ}$ at $0 \mathrm{~K}$ to $0^{\circ}$ at $30 \mathrm{~K}$. This contrasts with the results of Dionne (1976) who assumed a temperatureindependent canting of the rare earth spins, due to the strong anisotropy field. The anisotropy constant $K_{1}$, however is strongly temperature-dependent (Pearson 1962) and hence the canting angle is not likely to be temperature-independent. The observed variation of the canting angle with temperature is given in figure 3.

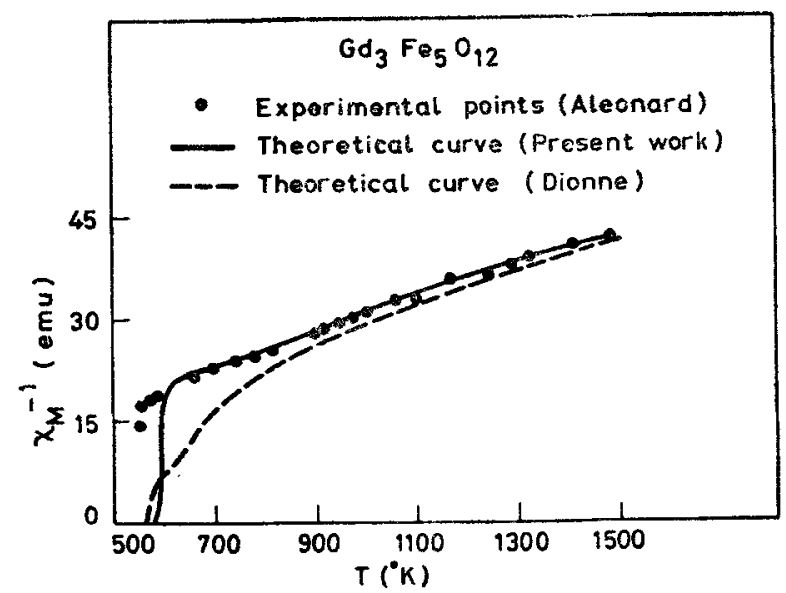

(a)

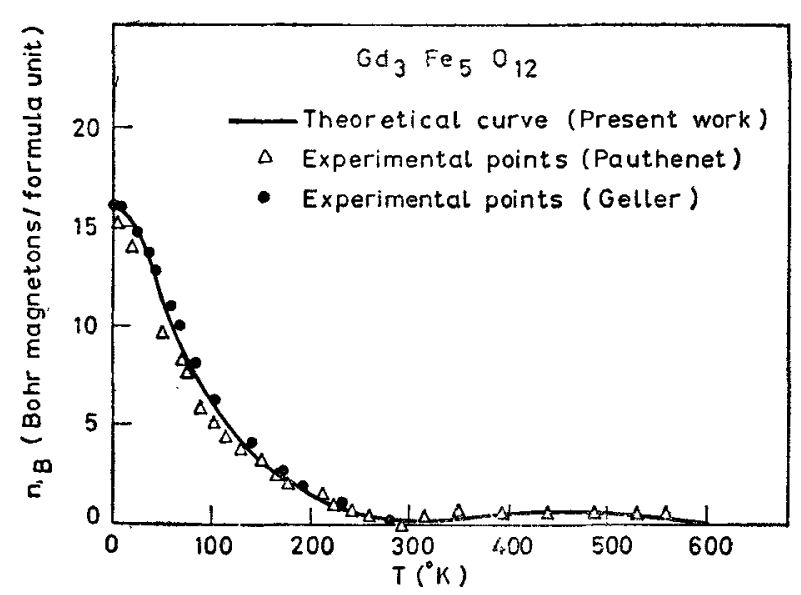

(b)

Figure 2. Theoretical and experimental curves for (a) saturation magnetisation $\left(M_{s}\right)$ and $(b)$ inverse susceptibility as a function of temperature for GdIG. The theoretical curve has been calculated using the exchange constants listed in table.1. 


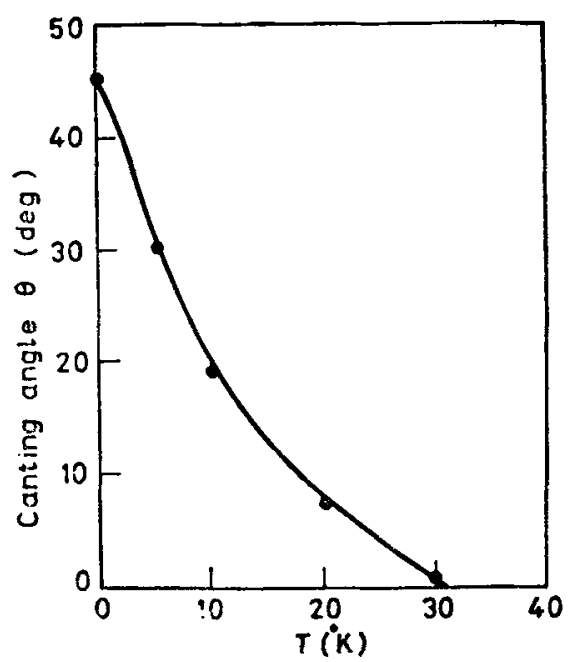

Figure 3. Variation of canting angle with temperature for Dy IG.

The fitting of the $M$, and $\chi^{-1}$ data for DyIG with temperature-dependent canting angle is given in figure 4 .

\section{Results and discussion}

The exchange constants for the three garnet systems listed in table 1 show that the magnitudes of the transition ion interactions, $J_{a z}, J_{a d}$ and $J_{d d}$ are greater than the transition ion-rare earth ion interaction as well as the rare earth-rare earth ion interaction. This is as expected on the basis of the overlap of the magnetic electrons on the wave functions of the ligands. On comparing the bond angles and distances of the exchange interactions in $\mathrm{Y}_{3} \mathrm{Fe}_{5} \mathrm{O}_{12}$ and $\mathrm{Fe}_{3} \mathrm{O}_{4}$ it is found that the $180^{\circ}$ a-d interaction in YIG is equivalent to the $180^{\circ} A-B$ interaction in $\mathrm{Fe}_{3} \mathrm{O}_{4}$ and the $90^{\circ} d-d$ interaction in YIG is equivalent to the $90^{\circ} A-A$ interaction in $\mathrm{Fe}_{3} \mathrm{O}_{4}$. Thus it is possible that the transfer integrals obtained for $\mathrm{Fe}_{3} \mathrm{O}_{4}$ by Srivastava et al (1979) can be used for YIG for the $180^{\circ} d^{5}-d^{5}$ interaction,

$$
J_{a d}=-\frac{1}{25}\left(\frac{2 b_{\sigma \sigma}^{2}}{U}+\frac{4 b^{2} \pi a}{U}\right) \text {. }
$$

The values of $b_{\sigma \sigma}, b_{\pi \pi}$ and $U$ for the spinels are $0.31 \mathrm{eV}, 0.18 \mathrm{eV}$ and $10 \mathrm{eV}$ respectively. This leads to a value of $-28 \mathrm{~K}$ for spinels which closely agrees with the value of $-30.4 \mathrm{~K}$ for the garnet systems given in table 1 . Similarly for the $90^{\circ} d^{5}-d^{5}$ interaction the value for the spinel ferrites is $-14 \mathrm{~K}$, which once again is close to the value of $-12.05 \mathrm{~K}$ obtained for garnets.

The ground state of $\mathrm{Dy}^{3+}$ has been studied by Grünberg et al (1969). There are a number of low lying excited states which can be occupied as the temperature is raised above $0 \mathrm{~K}$. The low temperature $(4 \cdot 2 \mathrm{~K})$ paramagnetic spectrum of Dy ${ }^{2+}$ in YAG and LAG observed by Ball et al (1963) shows a highly anisotropic 


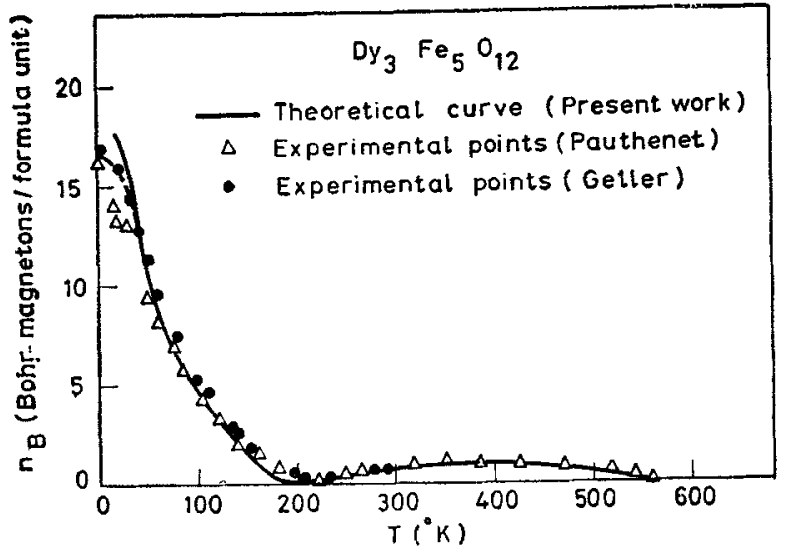

(a)

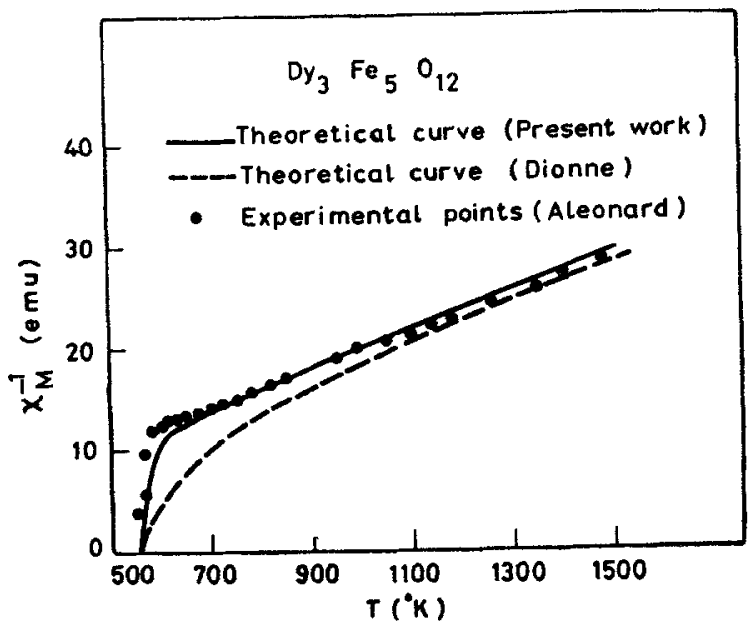

(b)

Figure 4. Theoretical and experimental curves for (a) saturation magnetisation $\left(M_{s}\right)$ and (b) inverse susceptibility as a function of temperature for Dy IG. The theoretical curve has been calculated using the exchange constants listed in table 1 with canting in the $c$-sublattice, the canting angle varying with temperature as shown in figure 3.

Table 1. Exchange constants in yttrium, gadolinium and dysprosium garnets (K).

\begin{tabular}{lcccccc}
\hline & $J_{a a}$ & $J_{a d}$ & $J_{d d}$ & $J_{a c}$ & $J_{d o}$ & $J_{c a}$ \\
\hline $\mathrm{Y}_{3} \mathrm{Fe}_{5} \mathrm{O}_{12}$ & -6.45 & -30.4 & -12.05 & 0 & 0 & 0 \\
$\mathrm{Gd}_{3} \mathrm{Fe}_{5} \mathrm{O}_{12}$ & -6.45 & -30.4 & -12.05 & -0.6 & -1.8 & 0 \\
$\mathrm{Dy}_{3} \mathrm{Fe}_{5} \mathrm{O}_{12}$ & -6.45 & -30.4 & -12.05 & -0.99 & -0.99 & 0.10 \\
\hline
\end{tabular}


behaviour. This indicates that the single ion anisotropy is large at low temperature, but as the temperature is raised on account of the occupancy of the low lying excited states, the anisotropy decreases sharply. This is supported by the observed behaviour of $K_{1}$ with temperature for YIG (Iida 1967) and DyIG (Pearson 1962). At $T=0$, the values of $K_{1}$ for YIG and DyIG are $-25 \times 10^{3}$ and $-2 \cdot 2 \times$ $10^{7} \mathrm{ergs} / \mathrm{cm}^{3}$, while at $T=77 \mathrm{~K}$ these values are $-22.4 \times 10^{3}$ and $-970 \times$ $10^{3} \mathrm{ergs} / \mathrm{cm}^{3}$, indicating that the single ion anisotropy of $\mathrm{Dy}^{3+}$ is highly temperature sensitive. The observed temperature dependence of the canting angle can therefore be interpreted to arise from the extreme anisotropy of the $\mathrm{Dy}^{3+}$ ion.

\section{Conclusions}

The exchange constants in YIG, GdIG and DyIG have been obtained from the magnetic data and interpreted in terms of the superexchange theory of Anderson.

\section{References}

Aléonard R 1960 J. Phys. Chem. Solids 15167

Anderson E E 1964 Phys. Rev. A134 1581

Anderson P W 1959 Phys. Rev. 1152

Ball M, Hutchins M T, Leask M J M and Wolf W P 1963 Proc. 8th Conf. Low Temp. Phys. 1962 (London: Butterworth) p. 248

Dionne G F 1970 J. Appl. Phys. 412264

Dionne G F 1976 J. Appl. Phys. 474220

Geller S, Williams H J, Sherwood R C, Remeika J P and Espinosa G P 1963 Phys. Rev. 1311080

Geller S, Remeika J P, Sherwood R C, Williams H J and Espinosa G P 1965 Phys. Rev. A137 1034

Grünberg P, Hüfner S, Orlich E and Schmitt J 1969 J. Appl. Phys. 401501

Iida S 1967 J. Phys. Soc. Jpn. 221201

Pauthenet $R 1957$ Les Propriétés magnétiques des ferrites d'yttrium de terres rares de formule $5 \mathrm{Fe}_{2} \mathrm{O}_{3} 3 \mathrm{M}_{2} \mathrm{O}_{3}$ Ph.D Thesis University, of Grenoble, France

Pearson R F 1962 J. Appl. Phys. 331236

Srivastava C M, Srinivasan G and Nanadikar N G 1979 Phys. Rev. 19499 